\title{
Delayed random walks: Investigating the interplay between delay and noise
}

\author{
Toru Ohira ${ }^{1}$ and John Milton ${ }^{2}$ \\ 1 Sony Computer Science Laboratories, Inc., Tokyo, Japan \\ ohira@csl.sony.co.jp \\ 2 Joint Science Department, The Claremont Colleges, Claremont, CA \\ jmilton@jsd.claremont.edu
}

Summary. A model for a 1-dimensional delayed random walk is developed by generalizing the Ehrenfest model of a discrete random walk evolving on a quadratic, or harmonic, potential to the case of non-zero delay. The Fokker-Planck equation derived from this delayed random walk (DRW) is identical to that obtained starting from the delayed Langevin equation, i.e. a first-order stochastic delay differential equation (SDDE). Thus this DRW and SDDE provide alternate, but complimentary ways for describing the interplay between noise and delay in the vicinity of a fixed point. The DRW representation lends itself to determinations of the joint probability function and, in particular, to the auto-correlation function for both the stationary and transient states. Thus the effects of delay are manisfested through experimentally measurable quantities such as the variance, correlation time, and the power spectrum. Our findings are illustrated through applications to the analysis of the fluctuations in the center of pressure that occur during quiet standing.

Key words: delay, random walk, stochastic delay differential equation, Fokker-Planck equation, auto-correlation function, postural sway

Feedback control mechanisms are ubiquitous in physiology $[2,8,17,22$, $34,41,47,48,60,63,65,66,67]$. There are two important intrinsic features of these control mechanisms: 1) all of them contain time delays; and 2) all of them are continually subjected to the effects of random, uncontrolled fluctuations (herein referred to as "noise"). The presence of time delays is a consequence of the simple fact that the different sensors that detect changes in the controlled variable and the effectors that act on this variable are spatially distributed. Since transmission and conduction times are finite, time delays are unavoidable. As a consequence, mathematical models for feedback control take the form of stochastic delay differential equations (SDDE); an example is the delayed Langevin equation or first-order SDDE with additive noise $[20,25,33,36,37,42,43,53,59]$

$$
d x(t)=-k x(t-\tau) d t+d W
$$


where $x(t), x(t-\tau)$ are, respectively, the values of the state variable at times $t$, and $t-\tau, \tau$ is the time delay, $k$ is a constant, and $W$ describes the Wiener process. In order to obtain a solution of (1) it is necessary to define an initial function, $x(t)=\Phi(t), t \in[-\tau, 0]$, denoted herein as $\Phi_{0}(t)$.

Understanding the properties of SDDEs is an important first step for interpreting the nature of the fluctuations in physiological variables measured experimentally $[11,37,53]$. However, an increasingly popular way to analyze these fluctuations has been to replace the SDDE by a delayed random walk, i.e. a discrete random walk for which the transition probabilities at the $n$-th step depend on the position of the walker $\tau$ steps before [55, 56, 57, 58, 59]. Examples include human postural sway [49,56], eye movements [46], neolithic transitions [18], econophysics [23, 58], and stochastic resonance-like phenomena [57]. What is the proper way to formulate the delayed random walk so that its properties are equivalent to those predicted by (1)?

An extensive mathematical literature has been devoted to addressing issues related to the existence and uniqueness of solutions of (1) and their stability $[51,52]$. These fundamental mathematical studies have formed the basis for engineering control theoretic studies of the effects of the interplay between noise and delay on the stability of man-made feedback control mechanisms $[6,54]$. Lost in these mathematical discussions of SDDEs is the nearly 100 years of careful experimental observation and physical insight that established the correspondence between (1) and an appropriately formulated random walk when $\tau=0[15,16,21,31,40,45,61]$ that does not have its counterpart for the case when $\tau \neq 0$. Briefly the current state of affairs is as follows. The continuous time model described by (1), referred to as the delayed Langevin equation, and the delayed random walk must be linked by a Fokker-Planck equation, i.e. a partial differential equation which describes the time evolution of the probability density function. This is because all of these models describe the same phenomenon and hence they must be equivalent in some sense. When $\tau=0$ it has been well demonstrated that the Langevin equation and the random walk leds to the same Fokker-Planck equation provided that the random walk occurs in a harmonic, or quadratic, potential (the Ehrenfest model) [31]. Although it has been possible to derive the Fokker-Planck equation from (1) when $\tau \neq 0[19,59]$, the form of the Fokker-Planck equation obtained from the delayed random walk has not yet been obtained. One of the objectives of this chapter is to show that the Fokker-Planck equation for the random walk can be readily obtained by generalizing the Ehrenfest model on a quadratic potential to non-zero delay. The importance of this demonstration is that it establishes that (1) and this delayed random walk give two different, but complimentary views of the same process.

Since (1) indicates that the dynamics observed at time $t$ depend on what happened at time $t-\tau$, it is obvious that the joint probability function must play a fundamental role in understanding the interplay between noise and delay. Moreover, the auto-correlation function, $c(\Delta) \equiv\langle x(t) x(t+\Delta)\rangle$, is essential for the experimental descriptions of real dynamical systems $[4,14,30]$. This 
follows from the fact that three measurements are required to fully describe a noisy signal: 1) its probability density function (e.g. uniform, Gaussian); 2) its intensity; and 3) its correlation time (e.g. white, colored). From a knowledge of $c(\Delta)$ we can obtain an estimate of the variance $(\Delta=0)$ which provides a measure of signal intensity, the correlation time, and the power spectrum. Armed with these quantities the experimentalist can directly compare experimental observation with prediction. Surprinsingly little attention has been devoted to the subject of the joint probability functions in the SDDE literature.

The organization of this chapter is as follows. First, we review the simple random walk that appears in standard introductory textbooks $[1,5,40,45,62]$. We use this simple random walk to introduce a variety of techniques that are used in the subsequent discussion including the concepts of the generating and characteristic functions, joint probability and the inter-relationship between the auto-correlation function and the power spectral density (WienerKhintchine theorem). The Fokker-Planck equation for the simple random walk is the familiar diffusion equation [45]. Second, we discuss the Ehrenfest model for a discrete random walk in a quadratic, or harmonic, potential in order to introduce the concept of stability into a random walk. Third, we introduce a discrete delay into the Ehrenfest model. The Fokker-Planck equation is obtained and is shown to be identical to that obtained starting from (1). In all cases particular attention is given to obtaining an estimate of $c(\Delta)$ and to demonstrating how the presence of $\tau$ influence the correlation time and the power spectrum. In the final section we review the application of delayed random walk models to the analysis of the fluctuations recorded during human postural sway.

\section{Simple random walk}

Analyses of random walks in its various forms lie at the core of statistical physics $[16,40,45,61,64]$ and its applications ranging from biology [5] to economics [44]. The simplest case describes a walker which is confined to move along a line by taking identical discrete steps at identical discrete time intervals (Figure 1). Let $X(n)$ be the position of the walker after the $n$-th step. Assume that at zero time all walkers start at the origin, i.e. the initial condition is $X(0)=0$, and that the probability, $p$, that the walker takes a step of unit length, $\ell$, to the right (i.e. $X(n+1)-X(n)=+\ell$ ) is the same as the probability, $q$, that it takes a step of unit length to the left (i.e. $X(n+1)-X(n)=-\ell)$, i.e. $p=q=0.5$.

The total displacement, $X$, after $n$ steps is

$$
X=\sum_{i=1}^{n} \ell_{i}
$$

where $\ell= \pm 1$. Since steps to the right and to the left are equally probable, then after a large number of steps, we have 


$$
\langle X\rangle=\sum_{i=1}^{n}\left\langle\ell_{i}\right\rangle=0
$$

where the notation $\langle\cdots\rangle$ signifies the "ensemble" average.
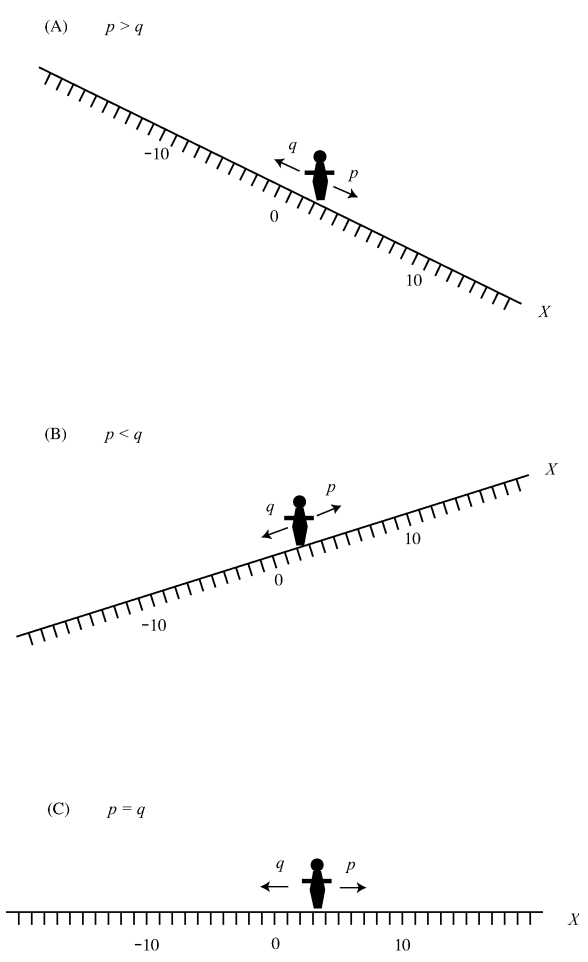

Fig. 1. Conceptual view of simple random walks. The probability to take a step to the right is $p$; a step to the left is $q$ : (A) $p>q$; (B) $p<q$, and (C) $p=q=0.5$.

Of course each time we construct, or realize, a particular random walk in this manner, the relationship given by (3) provides us no information as to how far a given walker is displaced from the origin after $n$ steps. One way to consider the displacement of each realization of a random walk is to compute $X^{2}$, i.e.

$$
\begin{aligned}
X^{2} & =\left(\ell_{1}+\ell_{2}+\cdots+\ell_{n}\right)\left(\ell_{1}+\ell_{2}+\cdots+\ell_{n}\right) \\
& =\sum_{i=1}^{n} \ell_{i}^{2}+\sum_{i \neq j}^{n} \ell_{i} \ell_{j}
\end{aligned}
$$

If we now average over many realizations of the random walk we obtain 


$$
\left\langle X^{2}\right\rangle=\sum_{i=1}^{n}\left\langle\ell_{i}^{2}\right\rangle+\sum_{i \neq j}^{n}\left\langle\ell_{i} \ell_{j}\right\rangle
$$

The first term is obviously $n \ell^{2}$. The second term vanishes since the direction of the step that a walker takes at a given instance does not depend on the direction of previous steps. In other words the direction of steps taken by the walker are uncorrelated and $\ell_{i}$ and $\ell_{j}$ are independent for $i \neq j$. Hence we have

$$
\left\langle X^{2}\right\rangle=n \ell^{2}
$$

The problem with this method of analysis of the random walk is that it is not readily transferable to more complex types of random walks. In particular, in order to use the notion of a random walk to investigate the properties of a stochastic delay differential equations, such as (1), it is necessary to introduce more powerful tools such as the characteristic, generating and auto-correlation functions. Without loss of generality we assume that the walker takes a step of unit length, i.e. $|\ell|=1$.

\subsection{Probability distribution function}

The probability that after $n$ steps the walker attains a position $r(X(n)=r)$ is $P(X=r, n)=P(r, n)$, where $P(r, n)$ is the probability distribution function and satisfies

$$
\sum_{r=-\infty}^{\infty} P(r, n)=1
$$

By analogy with the use of the characteristic function in continuous dynamical systems [7, 14], the discrete characteristic function,

$$
R(\theta, n)=\sum_{r=-\infty}^{+\infty} P(r, n) e^{j \theta r}
$$

where $\theta$ is the continuous "frequency" parameter, can be used to calculate $P(r, n)$. Since $R(\theta, n)$ is defined in terms of a Fourier series whose coefficients are the $P(r, n)$, the probability distribution after $n$ steps can be represented in integral form as

$$
P(r, n)=\frac{1}{2 \pi} \int_{-\pi}^{\pi} R(\theta, n) e^{-j \theta r} d \theta .
$$

In order to use $R(\theta, n)$ to calculate $P(r, n)$ we first write down an equation that describes the dynamics of the changes in $P(r, n)$ as a function of the number of steps, i.e.

$$
\begin{aligned}
& P(r, 0)=\delta_{r, 0} \\
& P(r, n)=p P(r-1, n-1)+q P(r+1, n-1) .
\end{aligned}
$$


where $\delta_{r, 0}$ is the Kronecker delta function defined by

$$
\begin{array}{ll}
\delta_{r, 0}=1, & (r=0) \\
\delta_{r, 0}=0, & (r \neq 0) .
\end{array}
$$

Second, we multiply both sides of (10) by $e^{j \theta r}$, and sum over $r$ to obtain

$$
\begin{aligned}
& R(\theta, 0)=1 \\
& R(\theta, n)=\left(p e^{j \theta}+q e^{-j \theta}\right) R(\theta, n-1) .
\end{aligned}
$$

The solution of these equations is

$$
R(\theta, n)=\left(p e^{j \theta}+q e^{-j \theta}\right)^{n}
$$

Taking the inverse Fourier transform of (11) we eventually obtain

$$
\begin{aligned}
P(r, n) & =\left(\begin{array}{c}
n \\
\frac{r+n}{2}
\end{array}\right) p^{\frac{r+n}{2}} q^{\frac{n-r}{2}}, \quad(r+n=2 m) \\
& =0, \quad(r+n \neq 2 m),
\end{aligned}
$$

where $m$ is a non-negative integer. For the special case that $p=q=0.5$ this expression for $P(r, n)$ simplifies to

$$
\begin{aligned}
P(r, n) & =\left(\begin{array}{c}
n \\
\frac{r+n}{2}
\end{array}\right)\left(\frac{1}{2}\right)^{n}, \quad(r+n=2 m) \\
& =0, \quad(r+n \neq 2 m),
\end{aligned}
$$

and we obtain

$$
\begin{aligned}
\langle X(n)\rangle & =0 \\
\sigma^{2}(n) & =n .
\end{aligned}
$$

When $p>q$ the walker drifts towards the right (Figure 1a) and when $p<q$ towards the left (Figure 1b). The evolution of $P(r, n)$ as a function of time when $p=0.6$ is shown in Figure 2 (for the special case of Brownian motion, i.e. $p=q=0.5$, see Figure 3).

\subsection{Variance}

The importance of $P(r, n)$ is that averaged quantities of interest, or expectations, such as the mean and variance can be readily determined from it. For a discrete variable, $X$, the moments can be determined by using the generating function, $Q(s, n)$, i.e.

$$
Q(s, n)=\sum_{r=-\infty}^{+\infty} s^{r} P(r, n)
$$




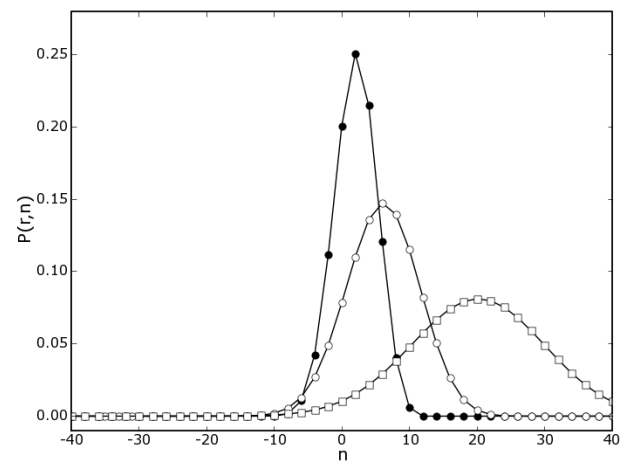

Fig. 2. The probability density function, $P(r, n)$, as a function of time for a simple random walk that starts at the origin with $p=0.6:(\bullet) n=10$, (०) $n=30,(\square)$ $n=100$. We have plotted $P(r, n)$ for even $r$ only: for these values of $n, P(r, n)=0$ when $r$ is odd.

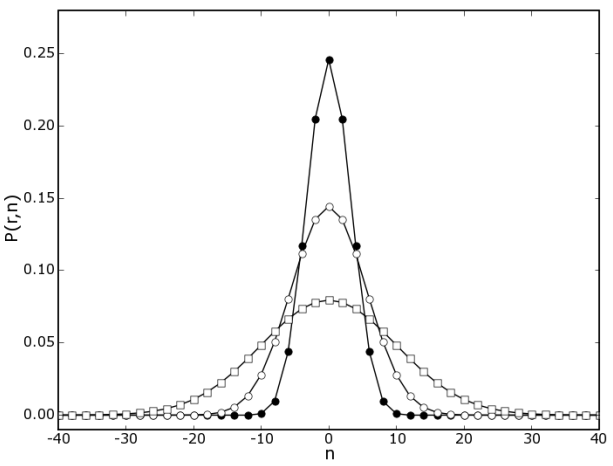

Fig. 3. The probability density function, $P(r, n)$, as a function of time for Brownian motion, i.e. a simple random walk that starts at the origin with $p=0.5:(\bullet) n=10$, (॰) $n=30,(\square) n=100$. We have plotted $P(r, n)$ for even $n$ only: for these values of $n, P(r, n)=0$ when $n$ is odd.

The averaged quantities of interest are calculated by differentiating $Q(s, n)$ with respect to $s$.

By repeating arguments analogous to those we used to obtain $P(r, n)$ from the characteristic function, we obtain

$$
Q(s, n)=\left[p s+\frac{q}{s}\right]^{n}
$$

The mean is obtained by differentiating $Q(s, n)$ with respect to $s$ 


$$
\left.\frac{\partial}{\partial s} Q(s, n)\right|_{s=1} \equiv\langle X(n)\rangle=n(p-q)
$$

The second differentiation leads to

$$
\left.\frac{\partial^{2}}{\partial s^{2}} Q(s, n)\right|_{s=1}=\left\langle X(n)^{2}\right\rangle-\langle X(n)\rangle
$$

The variance can be calculated from these two equations as $\sigma^{2}(n)=4 n p q$. The variance gives the intensity of the varying component of the random process, i.e. the $\mathrm{AC}$ component or the variance. The positive square root of the variance is the standard deviation which is typically referred to as the root-mean-square (rms) value of the AC component of the random process. When $\langle X(n)\rangle=0$, the variance equals the mean square displacement.

\subsection{Fokker-Planck equation}

We note here briefly that the random walks presented here has a correspondence with the continuous time stochastic partial differential equation

$$
\frac{d x}{d t}=\mu+\xi(t)
$$

where $\xi(t)$ is a gaussian white noise, and $\mu$ is a 'drift constant'. Both from the random walk presented above and from this differential equation, we can obtain the Fokker-Planck equation [21, 45]

$$
\frac{\partial}{\partial t} P(x, t)=-v \frac{\partial}{\partial x} P(x, t)+D \frac{\partial^{2}}{\partial x^{2}} P(x, t) .
$$

where $v$ and $D$ are constants. When there is no bias, $\mu=0, p=q=\frac{1}{2}$ and we have $v=0$, leading to the diffusion equation. This establishes a link between the Wiener process and simple symmetric random walk.

\subsection{Auto-correlation function: Special case}

The stationary discrete auto-correlation function, $C(\Delta)$, provides a measure of how much average influence random variables separated $\Delta$ steps apart have on each other. Typically little attention is given to determining the autocorrelation function for a simple random walk. However, it is useful to have a baseline knowledge of what the auto-correlation looks like for a simple random process.

Suppose we measure the direction that the simple random walker moves each step. Designate a step to the right as $R$, a step to the left as $L$, and a 'flip' as an abrupt change in the direction that the walker moves. Then the time series for a simple random walker takes the form 


$$
R \cdots \overbrace{R L}^{\text {flip }} \cdots \underbrace{L R}_{\text {flip }} \cdots \overbrace{R L}^{\text {flip }} \cdots
$$

Assume that the step interval, $\delta n$, is so small that the probability that two flips occur within the same step is approximately zero. The auto-correlation function, $C(\Delta)$, where $|\Delta| \geq|\delta n|$, for this process will be

$$
C(\Delta) \equiv\langle X(n) X(n+|\Delta|)\rangle=A^{2}\left(p_{0}(\Delta)-p_{1}(\Delta)+p_{2}(\Delta)-p_{3}(\Delta)+\cdots\right)
$$

where $p_{\kappa}(\Delta)$ is the probability that in a time interval $\Delta$ that exactly $\kappa$ flips occur and $A$ is the length of each step.

In order to calculate the $p_{\kappa}$ we proceed as follows. The probability that a flip occurs in $\delta n$ is $\lambda \delta n$, where $\lambda$ is some suitably defined parameter. Hence the probability that no flip occurs is $1-\lambda \delta n$. If $n>0$ then the state involving precisely $\kappa$ flips in the interval $(n, n+\delta n)$ arises from either $\kappa-1$ events in the interval $(0, n)$ with one flip in time $\delta n$, or from $\kappa$ events in the interval $(0, n)$ and no new flips in $\delta n$. Thus

$$
p_{\kappa}(n+\delta n)=p_{\kappa-1}(n) \lambda \delta n+p_{\kappa}(n)(1-\lambda \delta n)
$$

and hence we have

$$
\lim _{\delta n \rightarrow 0} \frac{p_{\kappa}(n+\delta n)-p_{\kappa}(n)}{\delta n} \equiv \frac{d p_{\kappa}(n)}{d n}=\lambda\left[p_{\kappa-1}(n)-p_{\kappa}(n)\right]
$$

for $\kappa>0$. When $\kappa=0$ we have

$$
\frac{d p_{0}(n)}{d n}=-\lambda p_{0}(n)
$$

and at $n=0$

$$
p_{0}(0)=1 \text {. }
$$

Equations (25)-(27) describe an iterative procedure to determine $p_{\kappa}$. In particular we have

$$
p_{\kappa}(\Delta)=\frac{(\lambda|\Delta|)^{\kappa} e^{-\lambda|\Delta|}}{\kappa !}
$$

The required auto-correlation function $C(\Delta)$ is obtained by combining $(23)$ and (28) as

$$
C(\Delta)=A^{2} e^{-2 \lambda|\Delta|}
$$

The auto-correlation function, $C(\Delta)$, and the power spectrum, $W(f)$, are intimately connected. In particular, they form a Fourier transform pair

$$
W(f)=\Delta \sum_{m=-(n-1)}^{n-1} C(\Delta) e^{-j 2 \pi f m \Delta}, \quad-\frac{1}{2 \Delta} \leq f<\frac{1}{2 \Delta}
$$

and 


$$
C(\Delta)=\int_{-1 / 2 \Delta}^{1 / 2 \Delta} W(f) e^{j 2 \pi f \Delta} d f, \quad-n \Delta \leq \Delta \leq n \Delta
$$

Our interest is to compare $C(\Delta)$ and $W(f)$ calculated for a discrete random walk to those that would be observed for a continuous random walk, respectively, $c(\Delta)$ and $w(f)$. Thus we assume in the discussion that follows that the length of the step taken by the random walker can be small enough so that we can replace (30) and (31) by, respectively,

$$
w(f)=\int_{-\infty}^{\infty} c(\Delta) e^{-j 2 \pi f \Delta} d \Delta
$$

and

$$
c(\Delta)=\int_{-\infty}^{\infty} w(f) e^{j 2 \pi f \Delta} d f
$$

Together (32) and (33) (or (30) and (31) are referred to as the WienerKhintchine theorem.

The power spectrum, $w(f)$, describes how the energy (or variance) of signal is distributed with respect to frequency. Since the energy must be the same whether we are in the time or frequency domian, it must necessarily be true that

$$
\int_{-\infty}^{\infty}|X(t)|^{2} d t=\int_{-\infty}^{\infty}|w(f)|^{2} d f
$$

This result is sometimes referred to as Parseval's formula. If $g(t)$ is a continuous signal, $w(f)$ of the signal is the square of the magnitude of the continuous Fourier transform of the signal, i.e.

$$
w(f)=\left|\int_{-\infty}^{\infty} g(t) e^{-j 2 \pi f t} d t\right|^{2}=|G(f)|^{2}=G(f) G^{*}(f)
$$

where $G(f)$ is the continuous Fourier transform of $g(t)$ and $G^{*}(f)$ is its complex conjugate.

Thus we can determine $w(f)$ for this random process as

$$
w(f)=\frac{2 A^{2}}{\lambda}\left[\frac{1}{1+(\pi f / \lambda)^{2}}\right]
$$

Figure 4 shows $c(\Delta)$ and $w(f)$ for this random process, where $f$ is $\Delta^{-1}$. We can see that the noise spectrum for this random process is essentially "flat" for $f \ll \lambda$ and thereafter decays rapidly to zero with a power law $1 / f^{2}$.

\subsection{Auto-correlation function: General case}

The important point is that the Fourier transform pairs given by (32)-(33) are valid whether $X(t)$ represents a deterministic time series or a realization of a stochastic process [30]. Unfortunately, it is not possible to reliably 

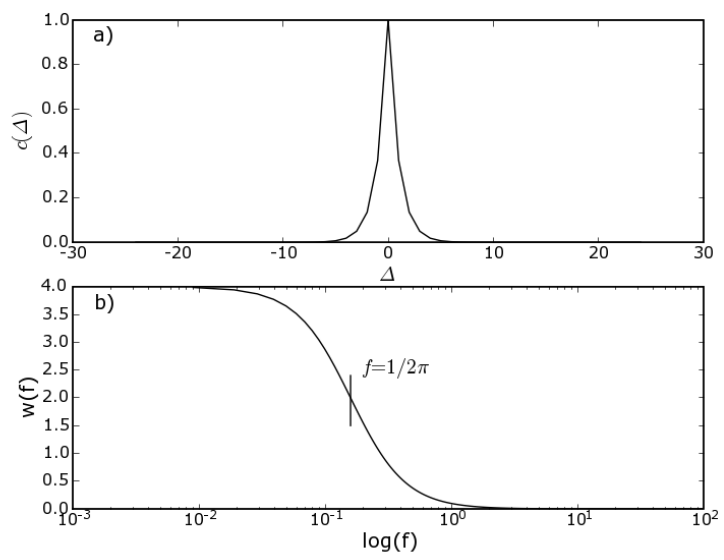

Fig. 4. (a) Auto-correlation function, $c(\Delta)$ and (b) power spectrum, $w(f)$, for the random process described by (22). Parameters: $A=1, \lambda=0.5$.

estimate $w(f)$ from a finitely long time series (see pp. 211-213 in [30]). The problem is that $w(f)$ measured for a finite length stochastic processes does not converge in any statistical sense to a limiting value as the sample length becomes infinitely long. This observation should not be particularly surprising. Fourier analysis is based on the assumption of fixed amplitudes, frequencies, and phases, but time series of stochastic processes are characterized by random changes of amplitudes, frequencies, and phases. Unlike $w(f)$ a reliable estimate of $c(\Delta)$ can be obtained from a long time series. These observations emphasize the critical importance of the Wiener-Khintchine theorem for describing the properties of a stochastic dynamical system in the frequency domain.

In order to define $C(\Delta)$ for a random walk it is necessary to introduce the concept of a joint probability, $P\left(r, n_{1} ; m, n_{2}\right)$. The joint probability is a probability density function that describes the occurence $X\left(n_{1}\right)=r$ and $X\left(n_{2}\right)=m$, i.e. the probability that $X\left(n_{1}\right)$ is at $r$ at $n_{1}$ when $X\left(n_{2}\right)$ is at site $m$ at $n_{2}$. The importance of $P\left(r, n_{1} ; m, n_{2}\right)$ is that we can use it to determine the probability that each of these events occurs separately. In particular, the probability, $P\left(r, n_{1}\right)$, that $X\left(n_{1}\right)$ is at site $r$ irrespective of the location of $X\left(n_{2}\right)$ is

$$
P\left(r, n_{1}\right)=\sum_{m=-\infty}^{+\infty} P\left(r, n_{1} ; m, n_{2}\right)
$$

Similarly, the probability, $P\left(m, n_{2}\right)$, that $X\left(n_{2}\right)$ is at site $m$ irrespective of the location of $X\left(n_{1}\right)$ is 


$$
P\left(m, n_{2}\right)=\sum_{r=-\infty}^{+\infty} P\left(r, n_{1} ; m, n_{2}\right)
$$

Using these definitions and the fact that $P\left(r, n_{1} ; m, n_{2}\right)$ is a joint probability distribution function, we can determine $C(\Delta, n)$ to be

$$
\begin{aligned}
C(\Delta, n) & \equiv\langle X(n) X(n-\Delta)\rangle \\
& =\sum_{r=-\infty}^{+\infty} \sum_{m=-\infty}^{+\infty} r m P(r, n ; m, n-\Delta)
\end{aligned}
$$

The stationary auto-correlation function can be defined by taking the long time limit as follows

$$
\begin{aligned}
C(\Delta) & \equiv\langle X(n) X(n-\Delta)\rangle_{s} \equiv \lim _{n \rightarrow \infty} C(\Delta, n) \\
& \left.=\lim _{n \rightarrow \infty} \sum_{r=-\infty}^{+\infty} \sum_{m=-\infty}^{+\infty} r m P(r, n ; m, n-\Delta)\right)
\end{aligned}
$$

\section{Random walks on a quadratic potential}

The major limitation for applying simple random walk models to questions related to feedback is that they lack the notion of stability, i.e. the resistance of dynamical systems to the effects of perturbations. In order to understand how stability can be incorporated into a random walk it is useful to review the concept of a potential function, $\phi(x)$, in continuous time dynamical systems. For $\tau=0$ the deterministic version of (1) can be written as [26]

$$
\dot{x}(t)=-k x(t)=-\frac{d \phi}{d x}
$$

and hence

$$
\phi(x)=\int_{0}^{x} g(s) d s=\frac{k x^{2}}{2}
$$

describes a quadratic, or harmonic, function. The bottom of this well corresponds to the fixed-point attractor $x=0$; the well to its basin of attraction. If $x(t)$ is a solution of (39) then

$$
\begin{aligned}
\frac{d}{d t} \phi(x(t)) & =\frac{d}{d x} \phi(x(t)) \cdot \frac{d}{d t} x(t) \\
& =-[g(x(t))]^{2} \leq 0
\end{aligned}
$$

In other words $\phi$ is always decreasing along the solution curves and in this sense is analogous to the "potential functions" in physical systems.

The urn model developed by Paul and Tatyana Ehrenfest showed how a quadratic potential could be incorporated into a discrete random walk [15, 
$31,32]$. The demonstration that the Fokker-Planck equation obtained for the Ehrenfest random walk is the same as that for Langevin's equation, i.e. (1), is due to M. Kac [31]. Here we derive the auto-correlation function, $C(\Delta)$, for the Ehrenfest random walk and the Langevin equation. The derivation of the Fokker-Planck equation for $\tau=0$ and $\tau \neq 0$ is identical. Therefore we present the Fokker-Planck equation for the Ehrenfest random walk as a special case of that for a delayed random walk in section 3.1.

By analogy with the above observations, we can incorporate the influence of a quadratic-shaped potential on a random walker by assuming that the transition probability towards the origin increases linearly with distance from the origin (of course up to a point). In particular the transition probability for the walker to move toward the origin increases linearly at a rate of $\beta$ as the distance increases from the origin up to the position $\pm a$ beyond which it is constant (since the transition probability is between 0 and 1). Equation (10) becomes

$$
\begin{aligned}
P(r, 0)= & \delta_{r, 0} \\
P(r, n)= & g(r-1) P(r-1, n-1) \\
& +f(r+1) P(r+1, n-1)
\end{aligned}
$$

where $a$ and $d$ are positive parameters, $\beta=2 d / a$, and

$$
\begin{aligned}
& f(x)=\left\{\begin{array}{l}
\frac{1+2 d}{2} x>a \\
\frac{1+\beta n}{2}-a \leq x \leq a \\
\frac{1-2 d}{2} x<-a
\end{array}\right. \\
& g(x)=\left\{\begin{array}{l}
\frac{1-2 d}{2} x>a \\
\frac{1-\beta n}{2}-a \leq x \leq a \\
\frac{1+2 d}{2} x<-a
\end{array}\right.
\end{aligned}
$$

where $f(x), g(x)$ are, respectively, the transition probabilities to take a step in the negative and positive directions at position $x$ such that

$$
f(x)+g(x)=1
$$

The random walk is symmetric with respect to the origin provided that

$$
f(-x)=g(x) \quad(\forall x) .
$$

We classify random walks by their tendency toward move towards the origin. The random walk is said to be attractive when

$$
f(x)>g(x) \quad(x>0) .
$$

and repulsive when

$$
f(x)<g(x) \quad(x>0) .
$$

We note that when 


$$
f(x)=g(x)=\frac{1}{2} \quad(\forall x),
$$

the general random walk given by (41) reduces to the simple random walk discussed Section 2. In this section we consider only the attractive case.

\subsection{Auto-Correlation function: Ehrenfest random walk}

Assume that with sufficiently large $a$, we can ignore the probability that the walker is outside of the range $(-\mathrm{a}, \mathrm{a})$. In this case, the probability distribution function $P(r, n)$ approximately satisfies the equation

$$
\begin{aligned}
P(r, n)= & \frac{1}{2}(1-\beta(r-1)) P(r-1, n-1) \\
& +\frac{1}{2}(1+\beta(r+1)) P(r+1, n-1) .
\end{aligned}
$$

By symmetry, we have

$$
\begin{aligned}
P(r, n) & =P(-r, n) \\
\langle X(t)\rangle & =0
\end{aligned}
$$

The variance, obtained by multiplying (46) by $r^{2}$ and summing over all $r$, is

$$
\sigma^{2}(n)=\left\langle X^{2}(n)\right\rangle=\frac{1}{2 \beta}\left(1-(1-2 \beta)^{n}\right)
$$

Thus the variance in the stationary state is

$$
\sigma_{s}^{2}=\left\langle X^{2}\right\rangle_{s}=\frac{1}{2 \beta} .
$$

The auto-correlation function for this random walk can be obtained by rewriting (46) in terms of joint probabilities to obtain

$$
\begin{aligned}
P(r, n ; m, n-\Delta)= & \frac{1}{2}(1-\beta(r-1)) P(r-1, n-1 ; m, n-\Delta) \\
& +\frac{1}{2}(1+\beta(r+1)) P(r+1, n-1 ; m, n-\Delta) .
\end{aligned}
$$

By defining

$$
P_{s}(r ; m, \Delta) \equiv \lim _{n \rightarrow \infty} P(r, n ; m, n-\Delta)
$$

the joint probability obtained in the stationary state, i.e. the long time limit, becomes

$$
\begin{aligned}
P_{s}(r ; m, \Delta)= & \frac{1}{2}(1-\beta(r-1)) P_{s}(r-1 ; m, \Delta-1) \\
& +\frac{1}{2}(1+\beta(r+1)) P_{s}(r+1 ; m, \Delta-1) .
\end{aligned}
$$


Multiplying by $r m$ and summing over all $r$ and $m$ yields

$$
C(\Delta)=(1-\beta) C(\Delta-1)
$$

which we can rewrite as

$$
C(\Delta)=(1-\beta)^{\Delta} C(0)=\frac{1}{2 \beta}(1-\beta)^{\Delta}
$$

where $C(0)$ is equal to the mean-square displacement, $\left\langle X^{2}\right\rangle_{s}$. When $\beta<<1$, we can approximate,

$$
C(\Delta) \approx \frac{1}{2 \beta} e^{-\beta|\Delta|} .
$$

Then, from the Wiener-Khintchine theorem we have

$$
w(f) \approx \frac{2}{\beta^{2}}\left[\frac{1}{1+(2 \pi f / \beta)^{2}}\right]
$$

These expressions for $C(\Delta)$ and $w(f)$ are in the same form as those obtained for a random process, i.e. (28) and (36). Hence $C(\Delta)$ and $w(f)$ are qualitatively the same as those shown in Figure 4.

\subsection{Auto-correlation function: Langevin equation}

When $\tau=0$, (1) describes the effects of random perturbations on a dynamical system confined to move within a quadratic potential. It is well known that the variance, $c(0)$, is

$$
c(0)=\frac{1}{2 k}
$$

which is identical to (53) if we identify $k$ with $\beta$ and take $\Delta=0[21,35]$.

In order to determine $c(\Delta)$ and $w(f)$ we note that when $\tau=0,(1)$ describes the effects of a low-pass filter on $\delta$-correlated (white) noise. Thus we can write the Fourier transform of (1) when $\tau=0$ as

$$
X(f)=H(f) I(f)
$$

where the frequency response, $H(f)$, is given by

$$
H(f)=\frac{1}{j 2 \pi f+k}
$$

and

$$
I(f)=\sigma^{2}
$$

where $\sigma$ is a constant. Hence we obtain

$$
w(f)=\frac{\sigma^{2}}{(2 \pi f)^{2}+k^{2}}
$$

and, applying the Weiner-Khintchine theorem,

$$
c(\Delta)=\frac{\sigma^{2}}{2 k} e^{-k \Delta}
$$

Equation (54) is the same as (59) and (53) is the same as (60). 


\section{Delayed random walks}

For a delayed random walk, the transition probability depends on its past state. If we generalize the random walk in a quadratic potential developed in Section 2 to non-zero delay we obtain the following definition for the transition probability

$$
\begin{aligned}
P(r, n+1)= & \sum_{m} g(m) P(r-1, n ; m, n-\tau) \\
& +\sum_{m} f(m) P(r+1, n ; m, n-\tau),
\end{aligned}
$$

where the position of the walker at time $n$ is $X(n), P(r, n)$ is the joint probability for the walker to be at $X(n)=r$ and $P(r, n ; m, n-\tau)$ is the joint probability such that $X(n)=r$ and $X(n-\tau)=m$ takes place. $f(x)$ and $g(x)$ are transition probabilities for the walker to take the step to the negative $(-1)$ and positive $(+1)$ directions respectively, and are the same as those used for the random walk in a quadratic potential described in the previous section.

\subsection{Delayed Fokker-Planck equation}

Here we derive the Fokker-Planck equation for the delayed random walk in a quadratic potential. This method is a direct use of the procedure used by M. Kac to obtain the Fokker-Planck equation for the case that $\tau=0$. For $f$ and $g$ as defined in the previous section, (61) becomes

$$
\begin{aligned}
P(r, n+1)= & \sum_{m} \frac{1}{2}(1-\beta m) P\left(r-1, n ; m, n-\tau_{d}\right) \\
& +\sum_{m} \frac{1}{2}(1+\beta m) P\left(r+1, n ; m, n-\tau_{d}\right),
\end{aligned}
$$

To make a connection, we assume that the random walk takes a step with a size of $\Delta x$ at a time interval of $\Delta t$, both of which are very small compared to the scale of space and time we are interested in. We take $x=r \Delta x$ and

$y=m \Delta x, t=n \Delta t$ and $\tau=\tau_{d} \Delta t$. With this stipulation, we can re-write $(62)$ as follows

$$
\begin{aligned}
& \frac{P(x, t+\Delta t)-P(x, t)}{\Delta t}= \\
& \frac{1}{2}\left(\frac{P(x-\Delta x, t)+P(x+\Delta x, t)-2 P(x, t)}{(\Delta x)^{2}}\right)\left(\frac{(\Delta x)^{2}}{\Delta t}\right) \\
& +\frac{1}{2} \sum_{\frac{y}{\Delta x}} \frac{y}{\Delta x}(P(x, t ; y, t-\tau)-P(x-\Delta x, t ; y, t-\tau)) \frac{\beta}{\Delta t} \\
& +\frac{1}{2} \sum_{\frac{y}{\Delta x}} \frac{y}{\Delta x}(P(x+\Delta x, t ; y, t-\tau)-P(x, t ; y, t-\tau)) \frac{\beta}{\Delta t}
\end{aligned}
$$


In the limits

$$
\begin{aligned}
& \Delta x \rightarrow 0, \quad \Delta t \rightarrow 0, \quad \beta \rightarrow 0 \\
& \frac{(\Delta x)^{2}}{2 \Delta t} \rightarrow D, \quad \frac{\beta}{\Delta t} \rightarrow \gamma, \quad n \Delta t \rightarrow t, \quad \tau_{d} \Delta t \rightarrow \tau \\
& n \Delta x \rightarrow x, \quad m \Delta x \rightarrow y,
\end{aligned}
$$

the difference equation (63) goes over to the following integro-partial differential equation

$$
\begin{aligned}
& \frac{\partial}{\partial t} \int_{-\infty}^{\infty} P(x, t ; y, t-\tau) d y= \\
& \quad \int_{-\infty}^{\infty} \gamma \frac{\partial}{\partial x}(y P(x, t ; y, t-\tau)) d y+D \int_{-\infty}^{\infty} \frac{\partial^{2}}{\partial x^{2}} P(x, t ; y, t-\tau) d y
\end{aligned}
$$

This is the same Fokker-Planck equation that is obtained from the delayed Langevin equation, (1) [19]. When $\tau=0$ we have

$$
P(x, t ; y, t-\tau) \rightarrow P(x, t) \delta(x-y),
$$

and the above Fokker-Planck equation reduces to the following familiar form.

$$
\frac{\partial}{\partial t} P(x, t)=\gamma \frac{\partial}{\partial x}(x P(x, t))+D \frac{\partial^{2}}{\partial x^{2}} P(x, t) .
$$

Thus, the correspondence between Ehrenfest's model and Langevin equation carries over to that between the delayed random walk model and the delayed Langevin equation.

We can re-write (65) by making the definition

$$
P_{\tau}(x, t) \equiv \int_{-\infty}^{\infty} P(x, t ; y, t-\tau) d y
$$

to obatin

$$
\frac{\partial}{\partial t} P_{\tau}(x, t)=\int_{-\infty}^{\infty} \gamma \frac{\partial}{\partial x}[y P(x, t ; y, t-\tau)] d y+D \frac{\partial^{2}}{\partial x^{2}} P_{\tau}(x, t)
$$

In this form we can more clearly see the effect of the delay on the drift of the random walker.

\subsection{Auto-correlation function: delayed random walk}

Three properties delayed random walks are particularly important for the discussion that follows. First, by the symmetry with respect to the origin, we have that the average position of the walker is 0 . In particular, for an attractive delayed random walk, the stationary state (i.e. when $n \rightarrow \infty$ ) 


$$
P(r, n+1 ; r+1, n)=P(r+1, n+1 ; r, n) .
$$

We can show the above as follows. By the definition of the stationarity, we have

$$
\begin{gathered}
P(r, n+1 ; r+1, n)+P(r, n+1 ; r-1, n)= \\
P(r+1, n+1 ; r, n)+P(r-1, n+1 ; r, n) .
\end{gathered}
$$

For $r=0$, we note that due to the symmetry, we have

$$
P(0, n+1 ; 1, n)=P(1, n+1 ; 0, n) .
$$

Using these two equations inductively leads us to the desired relation (69). Second, the generating function

$$
\begin{aligned}
& \langle\cos (\alpha X(n))\rangle= \\
& \cos (\alpha)\langle\cos (\alpha X(n))\rangle+\sin (\alpha)\langle\sin (\alpha X(n))\{f(X(n-\tau))-g(X(n-\tau))\}\rangle
\end{aligned}
$$

can be obtained by multiplying Eq. (61) for the stationary state by $\cos (\alpha r)$ and then summing over $r$ and $m$.

Finally we have the following invariant relationship with respect to the delay

$$
\frac{1}{2}=\langle X(n)\{f(X(n-\tau))-g(X(n-\tau))\}\rangle
$$

When we choose $f, g$ as before, this invariant relation in Eq. (74) becomes the following with this model:

$$
\langle X(n+\tau) X(n)\rangle=C(\tau)=\frac{1}{2 \beta} .
$$

This invariance with respect to $\tau$ of the correlation function with $\tau$ steps apart is a simple characteristic of this quadratic potential delayed random walk model. This property is a key to obtaining the analytical expression for the correlation function. Below we discuss $C(\Delta)$ for the stationary state. Observations pertaining to $C(\Delta)$ in the transient state are presented in Section 4.1.

For the stationary state and $0 \leq \Delta \leq \tau$, the following is obtained from the definition (61):

$$
\begin{aligned}
P_{s}(r, n+\Delta ; m, n) & =\sum_{\ell} g(\ell) P_{s}(r-1, n+\Delta ; m, n+1 ; \ell, n+\Delta-\tau) \\
& +\sum_{\ell} f(\ell) P_{s}(r+1, n+\Delta ; m, n+1 ; \ell, n+\Delta-\tau)
\end{aligned}
$$

We can derive the following equation for the correlation function by multiplication of this equation by $r m$ and summing over. 


$$
C(\Delta)=C(\Delta-1)-\beta C(\tau+1-\Delta), \quad(0 \leq \Delta \leq \tau)
$$

A similar argument can be given for $\tau<\Delta$,

$$
C(\Delta)=C(\Delta-1)-\beta C(\Delta-1-\tau), \quad(\tau<\Delta) .
$$

Equations (77) and (78) can be solved explicitly using (75). In particular, for $0 \leq \Delta \leq \tau$ we obtain

$$
\begin{aligned}
C(\Delta) & =C(0) \frac{\left(z_{+}^{\Delta}-z_{+}^{\Delta-1}\right)-\left(z_{-}^{\Delta}-z_{-}^{\Delta-1}\right)}{z_{+}-z_{-}}-\frac{1}{2} \frac{\left(z_{+}^{\Delta}-z_{-}^{\Delta}\right)}{z_{+}-z_{-}} \\
C(0) & =\frac{1}{2 \beta} \frac{\left(z_{+}-z_{-}\right)+\beta\left(z_{+}^{\tau}-z_{-}^{\tau}\right)}{\left(z_{+}^{\tau}-z_{+}^{\tau-1}\right)-\left(z_{-}^{\tau}-z_{-}^{\tau-1}\right)}
\end{aligned}
$$

where

$$
z_{ \pm}=\left(1-\frac{\beta^{2}}{2}\right) \pm \frac{\beta}{2} \sqrt{\beta^{2}-4}
$$

For $\tau<\Delta$, it is possible to write $C(\Delta)$ in a multiple summation form, though the expression becomes rather complex. For example, with $\tau<\Delta \leq$ $2 \tau$,

$$
C(\Delta)=\frac{1}{2 \beta}-\beta \sum_{i=1}^{\Delta-\tau} C(i)
$$

where the $C(i)$ are given by (79).
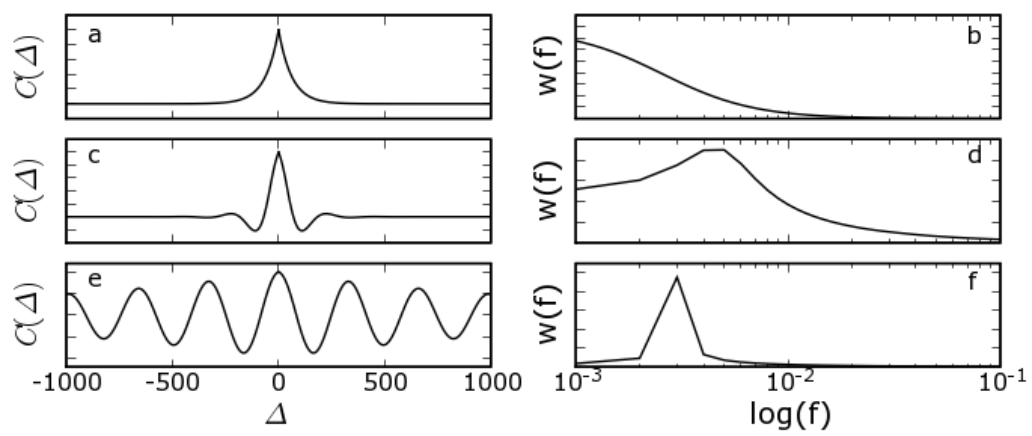

Fig. 5. Stationary auto-correlation function, $C(\Delta)$ (left-hand column) and power spectra, $W(f)$ (right-hand column) for an attractive delayed random walk on a quadratic potential for different time delays, $\tau$. Stationary correlation function $C(u)$ calculated using $(80)$ and $W(f)$ calculated from this using the Weiner-Klintchine theorem. The parameters were $a=50, d=0.4$ and $\tau$ was 0 for (a) and (b), 40 for (c) and (d) and 80 for (e) and (f). 
Figure 5 compares $C(\Delta)$ and $W(f)$ for different values of the delay. As we increase $\tau$, oscillatory behavior of the correlation function appears. The decay of the peak envelope is found numerically to be exponential. The decay rate of the envelope for the small $u$ is approximately $1 /(2 C(0))$.

\subsection{Auto-correlation function: delayed Langevin equation}

The statistical properties of (1) have been extensively studied previously [20, $25,33,42,43]$ Two approaches can be used to calculate $c(\Delta)$.

First, we can take the Fourier transform of (1) to determine $w(f)$ and then use the Wiener-Khintchine theorem to obtain $c(\Delta)$. From (56) we obtain the frequency response, $H(f)$,

$$
H(f)=\frac{1}{j 2 \pi f+\mu e^{-j 2 \pi f \tau}}
$$

As before we assume a "white noise" input and hence the power spectrum, $w(f)$, is

$$
\begin{aligned}
w(f) & =|H(f) \sigma|^{2} \\
& =\frac{\sigma^{2}}{(2 \pi f)^{2}-4 \pi f \mu \sin 2 \pi f \tau+\mu^{2}}
\end{aligned}
$$

Using the Wiener-Khintchine theorem we obtain

$$
c(\Delta)=\frac{\sigma^{2}}{2 \pi} \int_{0}^{\infty} \frac{\cos \omega \Delta}{\omega^{2}-2 \omega \mu \sin (\omega \tau)+\mu^{2}} d \omega
$$

where we have defined $\omega=2 \pi f$ to simplify the notation. The variance, $\sigma_{x}^{2}$ is equal to the value of $c(\Delta)$ when $\Delta=0$, i.e.

$$
\sigma_{x}^{2}=\frac{\sigma^{2}}{2 \pi} \int_{0}^{\infty} \frac{d \omega}{\mu^{2}+\omega^{2}-2 \mu \omega \sin (\omega \tau)}
$$

When (82) is integrated numerically the result obtained agrees with the results obtained by Küchler and Mensch (discussed below) for $0 \leq \Delta \leq \tau$ [25].

Second, Küchler and Mensch showed that the stationary correlation function for $\Delta<\tau$ could be obtained directly from (1) and was equal to

$$
c(\Delta)=c(0) \cos (\mu \Delta)-\frac{1}{2 \Delta} \sin (\mu \Delta)
$$

where

$$
c(0)=\frac{1+\sin (\mu \tau)}{2 \mu \cos (\mu \tau)}
$$

It should be noted that for small delay the variance increases linearly. This observation can be confirmed [25]. 
We now show that the expressions for $C(\Delta)$ obtained from the delayed random walk (80) is equivalent to those given by (83) for $0 \leq \Delta \leq \tau$. In particular, for small $\beta$, we have

$$
\begin{aligned}
\frac{\left(z_{+}^{\Delta}-z_{+}^{\Delta-1}\right)-\left(z_{-}^{\Delta}-z_{-}^{\Delta-1}\right)}{z_{+}-z_{-}} & \sim \cos (\beta \Delta) \\
\frac{\beta\left(z_{+}^{\Delta}-z_{-}^{\Delta}\right)}{z_{+}-z_{-}} & \sim \sin (\beta \Delta)
\end{aligned}
$$

Thus

$$
C(\Delta) \sim C(0) \cos (\beta \Delta)-\frac{1}{2 \Delta} \sin (\beta \Delta)
$$

with

$$
C(0) \sim \frac{1+\sin (\beta \tau)}{2 \beta \cos (\beta \tau)}
$$

\section{Postural sway}

Postural sway refers to the fluctuations in the center of pressure (COP) that occur as a subject stands quietly with eyes closed on a force platform $[13,53]$. Mathematical models for balance control identify three essential components $[17,49,50,56]: 1)$ feedback, 2) time delays, and 3) the effects of random, uncontrolled perturbations ("noise"). Thus it is not surprising that the first application of a delayed random walk was to investigate the fluctuations in COP [56]. In this study it was assumed that the probability $p_{+}(n)$ for the walker to take a step at time $n$ to the right (positive direction) was given by

$$
p_{+}(n)= \begin{cases}p & X(n-\tau)>0 \\ 0.5 & X(n-\tau)=0 \\ 1-p & X(n-\tau)<0\end{cases}
$$

where $0<p<1$. The origin is attractive when $p<0.5$. By symmetry with respect to the origin we have $\langle X(n)\rangle=0$. As shown in Figure 6 this simple model was remarkably capable of reproducing some of the features observed for the fluctuations in the center-of-pressure observed for certain human subjects.

There are a number of interesting properties of this random walk (Figure 7). First, for all choices of $\tau \geq 0, \sqrt{\left\langle X^{2}(n)\right\rangle}$ approaches a limiting value, $\Psi$. Second, the qualitative nature of the approach of $\sqrt{\left\langle X^{2}(n)\right\rangle}$ to $\Psi$ depends on the value of $\tau$. In particular, for short $\tau$ there is a non-oscillatory approach to $\Psi$, whereas for longer $\tau$ damped oscillations occur (Figure 7) whose period is approximately twice the delay. Numerical simulations of this random walk led to the approximation

$$
\Psi(\tau) \sim(0.59-1.18 p) \tau+\frac{1}{\sqrt{2}(1-2 p)}
$$



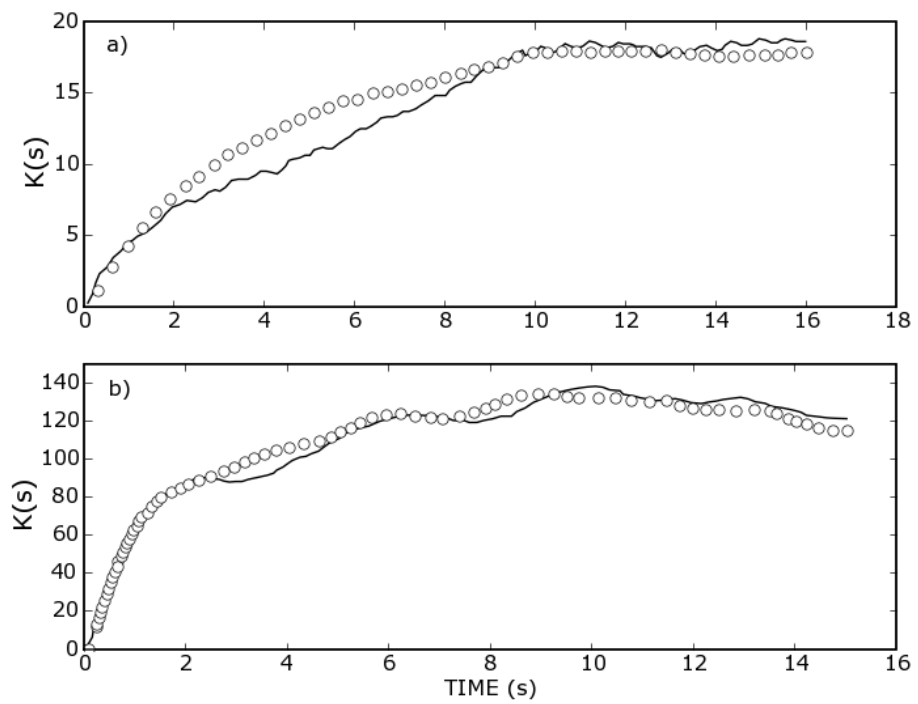

Fig. 6. Comparison of the two-point correlation function, $K(s)=\langle(X(n)-X(n-$ $\left.s))^{2}\right\rangle$, for the fluctuations in the center-of-pressure observed for two healthy subjects (solid line) with that predicted using a delayed random walk model (o). In a) the parameters for the delayed random walk were $p=0.35$ and $\tau=1$ with an estimated unit step length of $1.2 \mathrm{~mm}$ and a unit time of $320 \mathrm{~ms}$. In b) the parameters were $p=0.40$ and $\tau=10$ with an estimated step length and unit time step of, respectively, $1.4 \mathrm{~mm}$ and $40 \mathrm{~ms}$. For more details see [56].

This approximation was used to fit the delayed random walk model to the experimentally measured fluctuations in postural sway shown in Figure 6.

In the context of a generalized delayed random walk (61) introduced in Section $4,(87)$ corresponds to choosing $f(x)$ and $g(x)$ to be

$$
\begin{aligned}
& f(x)=\frac{1}{2}[1+\eta \theta(x)] \\
& g(x)=\frac{1}{2}[1-\eta \theta(x)]
\end{aligned}
$$

where

$$
\eta=1-2 p
$$

and $\theta$ is a step-function defined by

$$
\theta(x)= \begin{cases}1 & \text { if } x>0 \\ 0 & \text { if } x=0 \\ -1 & \text { if } x<0\end{cases}
$$

In other words the delayed random walk occurs on a V-shaped potential (which, of course is simply a linear approximation to a quadratic potential). 


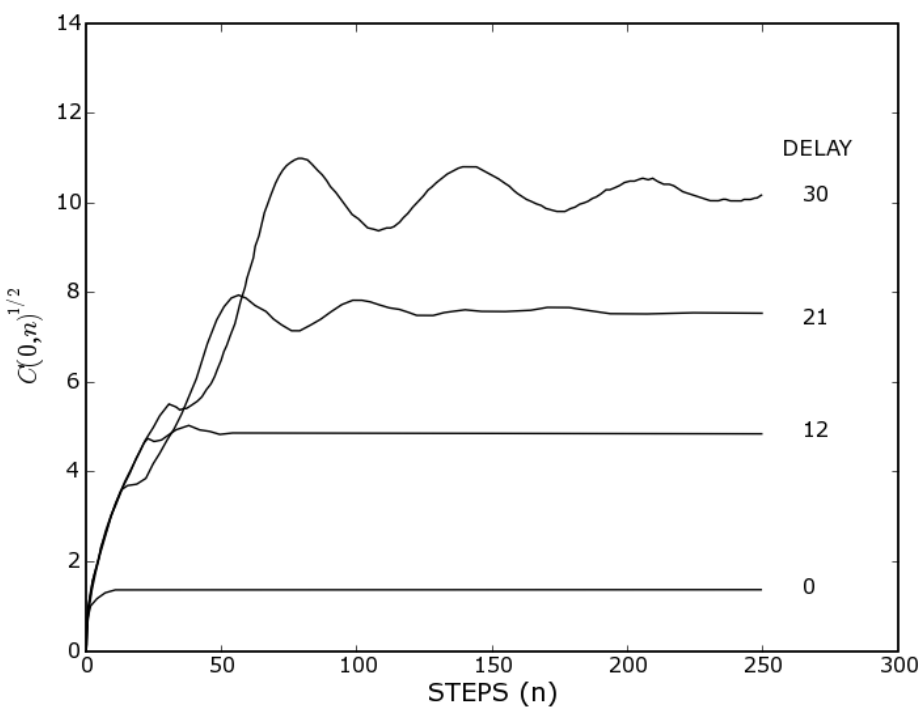

Fig. 7. Examples of dynamics of the root mean square position $C(0, t)^{1 / 2}$ for various choices of $\tau$ when $p=0.25$.

Below we briefly describe the properties of this delayed random walk (for more details see [59]).

By using symmetry arguments it can be shown that the stationary probability distributions $P_{s}(X)$ when $\tau=0$ can be obtained by solving the system of equations with the long time limit.

$$
\begin{aligned}
P(0, n+1) & =2(1-p) P(1, n) \\
P(1, n+1) & =\frac{1}{2} P(0, n)+(1-p) P(2, n) \\
P(r, n+1) & =p P(r-1, n)+(1-p) P(r+1, n) \quad(2 \leq r)
\end{aligned}
$$

where $P(r, n)$ is the probability to be at position $r$ at time $n$, using the trial function $P_{s}(r)=Z^{r}$, where

$$
P_{s}(r)=\lim _{n \rightarrow \infty} P(r, n)
$$

In this way we obtain

$$
\begin{aligned}
& P_{s}(0)=2 C_{0} p \\
& P_{s}(r)=C_{0}\left(\frac{p}{1-p}\right)^{r} \quad(1 \leq r)
\end{aligned}
$$

where 


$$
C_{0}=\frac{(1-2 p)}{4 p(1-p)}
$$

Since we know the p.d.f. we can easily calculate the variance when $\tau=0$, $\sigma^{2}(0)$, as

$$
\sigma^{2}(0)=\frac{1}{2(1-2 p)^{2}}
$$

The stationary probability distributions when $\tau>0$ can be obtained by solving the set of equations with the long time limit.

for $(0 \leq r<\tau+2)$

$$
\begin{aligned}
P(r, n+1)= & p P(r-1, n ; r>0, n-\tau)+\frac{1}{2} P(r-1, n ; r=0, n-\tau) \\
& +(1-p) P(r-1, n ; r<0, n-\tau)+p P(r+1, n ; r<0, n-\tau) \\
& +\frac{1}{2} P(r+1, n ; r=0, n-\tau)+(1-p) P(r+1, n ; r>0, n-\tau)
\end{aligned}
$$

for $(\tau+2 \leq r)$

$$
P(r, n+1)=p P(r-1, n)+(1-p) P(r+1, n)
$$

These equations are very tedious to solve and not very illuminating. Indeed we have only been able to obtain the following results for $\tau=1$

$$
\left\langle X^{2}\right\rangle=\frac{1}{2(1-2 p)^{2}}\left(\frac{7-24 p+32 p^{2}-16 p^{3}}{3-4 p}\right)
$$

and for $\tau=2$

$$
\left\langle X^{2}\right\rangle=\frac{1}{2(1-2 p)^{2}}\left(\frac{25-94 p+96 p^{2}+64 p^{3}-160 p^{4}+64 p^{5}}{5+2 p-24 p^{2}+16 p^{3}}\right)
$$

\subsection{Transient auto-correlation function}

In Section 4 we assumed that the fluctuations in COP were realizations of a stationary stochastic dynamical system. However, this assumption is by no means clear. An advantage of a delayed random walk model is that it is possible to gain some insight into the nature of the auto-correlation function for the transient state, $C_{t}(\Delta)$. In particular, for the transient state we can calculate in the similar manner to (77)-(78), the set of coupled dynamical equations

$$
\begin{aligned}
C_{t}(0, n+1) & =C_{t}(0, n)+1-2 \beta C_{t}(\tau, n-\tau) \\
C_{t}(\Delta, n+1) & =C_{t}(\Delta-1, n+1)-\beta C_{t}(\tau-(\Delta-1), n+\Delta-\tau), \quad(1 \leq \Delta \leq \tau) \\
C_{t}(\Delta, n+1) & =C_{t}(\Delta-1, n+1)-\beta C_{t}((\Delta-1)-\tau, n+1), \quad(\Delta>\tau)
\end{aligned}
$$


For the initial condition, we need to specify the correlation function for the interval of initial $\tau$ steps. When the random walker begins at the origin we have a simple symmetric random walk for $n \in(1, \tau)$. This translates to the initial condition for the correlation function as

$$
C_{t}(0, n)=n \quad(0 \leq \Delta \leq \tau) \quad C_{t}(u, 0)=0 \quad(\forall u) .
$$

The solution can be iteratively generated using (95) and this initial condition. We have plotted some examples for the dynamics of the mean square displacement $C_{t}(0, n)$ in Figure 8. Again, the oscillatory behavior arises with increasing $\tau$. Hence, the model discussed here shows the oscillatory behavior with increasing delay which appears in both its stationary and transient states.

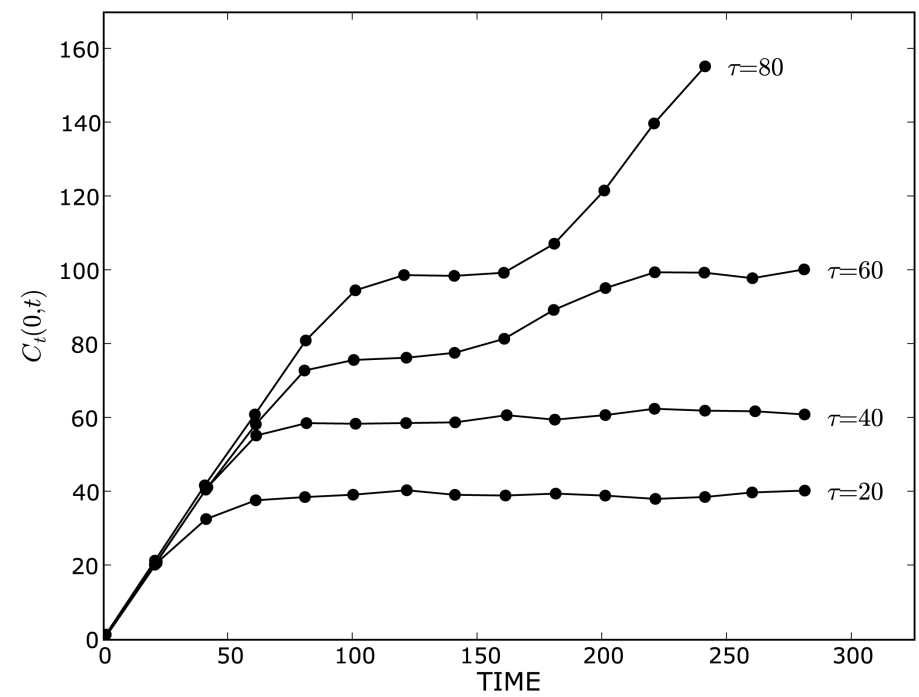

Fig. 8. Examples of dynamics of the transient variance $C_{t}(0, t)$ for different delays $\tau$. Data averaged from $10,000 \operatorname{simulations}(\bullet)$ is compared to that determined analytically from (95) (solid line). The parameters were $a=50, d=0.45$.

We also note that from (95), we can infer the corresponding set of equations for the transient auto-correlation function of (1) with a continuous time, $c_{t}(\Delta)$. They are given as follows.

$$
\frac{\partial}{\partial t} c_{t}(0, t)=-2 k c_{t}(\tau, t-\tau)+1
$$




$$
\begin{aligned}
\frac{\partial}{\partial \Delta} c_{t}(\Delta, t) & =-k c_{t}(\tau-\Delta, t+\Delta-\tau) \quad(0<\Delta \leq \tau) \\
\frac{\partial}{\partial \Delta} c_{t}(\Delta, t) & =-k c_{t}(\Delta-\tau, t) \quad(\tau<\Delta)
\end{aligned}
$$

Studies on these coupled partial differential equations with delay are yet to be done.

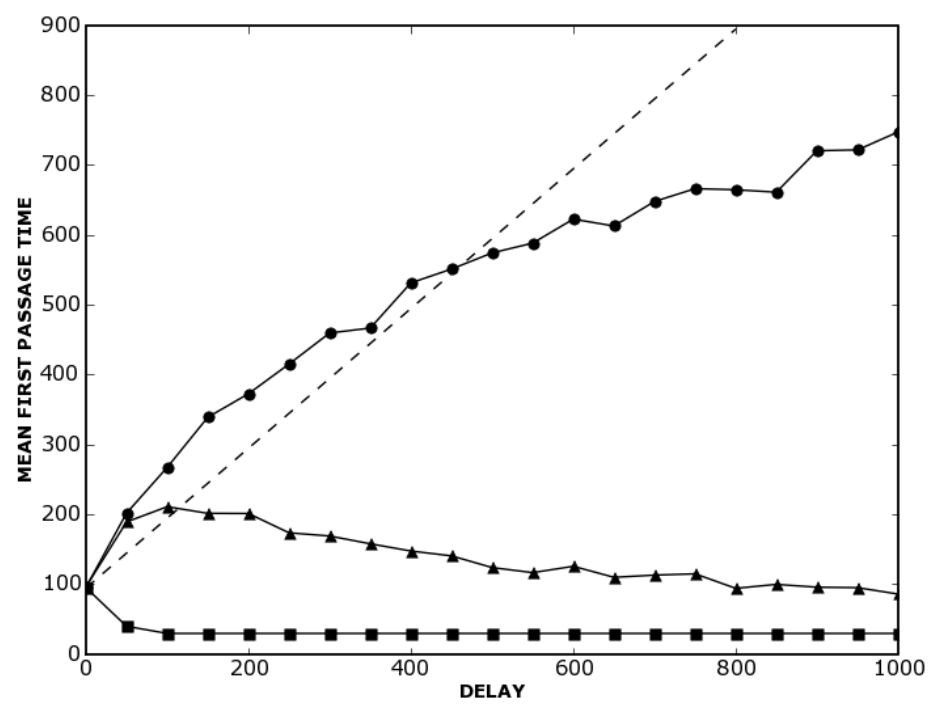

Fig. 9. The mean first passage time, $\hat{L}$, for a repulsive delayed random walk as a function of $\tau$ for three different choices of the initial fuction, $\left.\Phi_{0}(t): 1\right)$ a constant zero function (solid line, $\bullet$ ); 2) an initial function constructed from a simple random walk with $\tau=0$ (solid line, $\mathbf{\Delta}$ ); 3) a linear decreasing initial function with end points $x=\tau$ at $t=-\tau$ and $x=0$ at $t=0$ (solid line, $\mathbf{\square}$ ). In all cases $\Phi_{0}(0)=0$. The dashed line is equal to $\hat{L}_{\tau=0}+\tau$. For each choice of $\Phi_{0}(t), 500$ realizations were calculated with $X^{*}= \pm 30$ with $d=0.4$ and $a=30$.

\subsection{Balance control with positive feedback}

Up to this point we have assumed that the feedback for balance control is continuous and negative. However, careful experimental observations for postural sway $[38,39]$ and stick balancing at the fingertip $[11,12,28]$ indicate that the feedback is on average positive and that it is administered in a pulsatile, or ballistic, manner. Recently the following switch-type discontinuous model for postural sway that incorporates positive feedback has been introduced in an attempt to resolve this paradox $[49,50]$ : 


$$
\frac{d x}{d t}= \begin{cases}\alpha x(t-\tau)+\eta^{2} \xi(t)+K & \text { if } x(t-\tau)<-\Pi \\ \alpha x(t-\tau)+\eta^{2} \xi(t) & \text { if }-\Pi \leq x(t-\tau) \leq \Pi \\ \alpha x(t-\tau)+\eta^{2} \xi(t)-K & \text { if } x(t-\tau)>\Pi\end{cases}
$$

where $\alpha, K$, and $\Pi$ are positive constants. This model is a simple extension of a model proposed by Eurich and Milton [17] and states that the nervous system allows the controlled variable to drift under the effects of noise $(\xi(t))$ and positive feedback $(\alpha>0)$ with corrective actions ('negative feedback') taken only when $x(t-\tau)$ exceeds a certain threshold, $\Pi$. It is assumed that $\alpha$ is small and that $\tau<3 \pi / 2 \alpha$. This means that there is one real positive eigenvalue and an infinite number of complex eigenvalue pairs whose real part is negative. The magnitude of the real positive eigenvalue decreases as $\tau$ increases for $0<\tau<3 \pi / 2 \alpha$. "Safety net" type controllers also arise in the design of strategies to control attractors that have shallow basins of attraction $[24]$.

The cost to operate this discontinuous balance controller is directly proportional to the number of times it is activated. Thus the mathematical problem becomes that of determining the first passage times for a repulsive delayed random walk, i.e. the times it takes a walker starting at the origin to cross the threshold, $\pm|\Pi|$. Numerical simulations of a repulsive delayed random walk indicate that the mean first passage time depends on the choice of $\Phi_{0}(t)$ (see Figure 9).

In principle, the most probable, or mean, first passage time can be calculated from the backward Kolmogorov equation for (1). We have been unable

to complete this calculation. However, simulations of (97) indicate that this dicontinuous balance controller takes advantage of two intrinsic properties of a repulsive delayed random walk: 1) the time delay which slows scape, and 2 ) the fact that the distribution of first passage times is bomodal. Since the distribution of first passage times is biomodal it is possible that a reset due to the activation of the negative feedback controller can lead to a trasient confinement of the walker near the origin thus further slowing escape.

\section{Concluding remarks}

In this chapter we have shown that a properly formulated delayed random walk can provide an alternate and complimentary approach for the analysis for stochastic delay differential equations such as (1). By placing the study of a stochastic differential equation into the context of a random walk it is possible to draw upon the large arsenal of analytical tools previously developed for the study of random walks and apply them to the study of these complex dynamical systems. The advantages of this approach to the analysis of SDDEs include 1) it avoids issues inherent in the Ito versus Stratonovich stochastic calculus, 2) it provides insight into transient dynamics, and 3) it is, in principle, applicable to the study of delayed stochastic dynamical systems in the setting of complex potential surfaces such as those that arise in 
the setting of multistability. In general the use of the delayed random walk involves solutions in the form of equations which must be solved iteratively. However, this procedure causes no practical problem given the ready availability of symbolic manipulation computer software programs such as Maple ${ }^{\complement}$ and Mathematica ${ }^{(C)}$. The use of these computer programs compliment the variety of numerical methods $[3,27,29]$ that have been developed to investigate SDDEs, such as (1). However, much remains to be done, particularly the important cases in which the noise enters the dynamics in a multiplicative, or parametric, fashion $[9,10,52]$. Thus we anticipate that the subject of delayed random walks will continue to gain in importance.

\section{Acknowledgements}

We thank Sue Ann Campbell for useful discussions. We acknowledge support from the William R. Kenan, Jr. Foundation (JM) and the National Science Foundation (Grant 0617072)(JM,TO).

\section{References}

1. Bailey, N. T., The Elements of Stochastic Processes, J. Wiley \& Sons, New York, 1990.

2. Bechhoefer, J., 'Feedback for physicists: A tutorial essay on control', Rev. Mod. Phys. 77, 2005, 783-836.

3. Bellen, A. and Zennaro, M., Numerical Methods for Delay Differential Equations, Oxford University Press, New York, 2003.

4. Bendat, J. S. and Piersol, A. G., Random Data: Analysis and Measurement Procedures, 2nd Edition, J. Wiley \& Sons, New York, 1986.

5. Berg, H. C., Random Walks in Biology, expanded edition, Princeton University Press, Princeton, New Jersey, 1993.

6. Boukas, E-K. and Liu Z-K., Deterministic and Stochastic Time Delay Systems, Birkhäuser, Boston, 2002.

7. Bracewell, R. N., The Fourier Transform and Its Applications, 2nd edition, McGraw-Hill, New York, 1986.

8. Bratsun, D., Volfson, D., Tsimring, L. S. and Hasty, J., 'Delay-induced stochastic oscillations in gene regulation', Proc. Natl. Acad. Sci. USA 102, 2005, 14593-14598.

9. Cabrera, J. L. and Milton, J. G., 'On-off intermittency in a human balancing task', Phys. Lett. Rev. 89, 2002, 158702.

10. Cabrera, J. L. and Milton, J. G., 'Human stick balancing: Tuning Lévy flights to improve balance control', Chaos 14, 2004, 691-698.

11. Cabrera, J. L., Bormann, R., Eurich, C. W., Ohira, T. and Milton, J., 'Statedependent noise and human balance control', Fluctuation Noise Letters 4, 2004, L107-L117.

12. Cabrera, J. L., Luciani, C., and Milton, J., 'Neural control on multiple time scales: Insights from human stick balancing', Condensed Matter Physics 2, 2006, 373-383 
13. Collins, J. J. and De Luca, C. J., 'Random walking during quiet standing', Phys. Rev. Lett. 73, 1994, 907-912.

14. Davenport, W. B. and Root, W. L., An Introduction to the Theory of Random Signals and Noise, IEEE Press, New York, 1987.

15. Ehrenfest, P. and Ehrenfest, T., 'Über zwei bekannte Einwände gegan das Boltzmannsche H-Theorem', Phys. Zeit. 8, 1907, 311-314.

16. Einstein, A., 'Zür Theorie der Brownschen Bewegung', Annalen der Physik 19, 1905, 371-381.

17. Eurich, C. W. and Milton, J. G., 'Noise-induced transitions in human postural sway', Phys. Rev. E 54, 1996, 6681-6684.

18. Fort, J., Jana, D. and Humet, J., 'Multidelayed random walk: Theory and application to the neolithic transition in Europe', Phys. Rev. E. 70, 2004, 031913.

19. Frank, T. D., 'Delay Fokker-Planck equations, Novikov's theorem, and Boltzmann distributions as small delay approximations', Phys. Rev. E 72, 2005, 011112 .

20. Frank, T. D. and Beek, P. J., 'Stationary solutions of linear stochastic delay differential equations: Applications to biological systems', Phys. Rev. E 64, 2001, 021917.

21. Gardiner, C. W., Handbook of Stochastic Methods for Physics, Chemistry and the Natural Sciences, Springer-Verlag, New York, 1994.

22. Glass, L. and Mackey, M. C., From Clocks to Chaos: The Rhythms of Life, Princeton University Press, Princeton, New Jersey, 1988.

23. Grassia, P. S., 'Delay, feedback and quenching in financial markets', Eur. Phys. J. $B$ 17, 2000, 347-362.

24. Guckhenheimer, J., 'A robust hybrid stabilization strategy for equilibria', IEEE Trans. Automatic Control 40, 1995, 321-326.

25. Guillouzic, S., L'Heureux, I. and Longtin, A., 'Small delay approximation of stochastic delay differential equation', Phys. Rev. E 59, 1999, 3970-3982.

26. Hale, J. and Koçak, H., Dynamics and Bifurcations, Springer-Verlag, New York, 1991.

27. Hofmann, N. and Müller-Gronbach, T., 'A modified Milstein scheme for approximation of stochastic delay differential equation with constant time lag', $J$. Comput. Appl. Math. 197, 2006, 89-121.

28. Hosaka, T., Ohira, T., Luciani, C., Cabrera, J. L. and Milton, J. G., 'Balancing with noise and delay', Prog. Theoret. Physics Supple. 161, 2006, 314-319.

29. Hu, Y., Mohammed, S.-E. A. and Yan,F., 'Discrete time approximations of stochastic delay equations: the Milstein scheme', Ann. Probab. 32, 2004, 265314 .

30. Jenkins, G. M. and Watts, D. G., Spectral Analysis and its Applications, Holden-Day, San Francisco, 1968.

31. Kac, M., 'Random walk and the theory of Brownian motion', Amer. Math. Monthly 54, 1947, 369-391.

32. Karlin, S. and McGregor, J., 'Ehrenfest urn models', J. Appl. Prob. 2, 1965, $352-376$.

33. Küchler, U. and Mensch, B., 'Langevins stochastic differential equation extended by a time-delayed term', Stochastic Stochastic Rep. 40, 1992, 23-42.

34. Landry, M., Campbell, S. A., Morris, K. and Aguilar, C. O., 'Dynamics of an inverted pendulum with delayed feedback control', SIAM J. Dynam. Sys. 4, 2005, 333-351. 
35. Lasota, A. and Mackey, M. C., Chaos, Fractals and Noise: Stochastic Aspects of Dynamics, Springer-Verlag, New York, 1994.

36. Longtin, A., 'Noise-induced transitions at a Hopf bifurcation in a first-order delay-differential equation', Phys. Rev. A 44, 1991, 4801-4813.

37. Longtin, A., Milton, J. G., Bos, J. E., and Mackey, M. C., 'Noise and critical behavior of the pupil light reflex at oscillation onset', Phys. Rev. A 41, 1990, 6992-7005.

38. Loram, I. D. and Lakie, M., 'Human balancing of an inverted pendulum: position control by small, ballistic-like, throw and catch movements', J. Physiol. 540, 2002, 1111-1124.

39. Loram, I. D., Maganaris,C. N. and Lakie, M., 'Active, non-spring-like muscle movements in human postural sway: how might paradoxical changes in muscle length be produced?' J. Physiol. 564.1, 2005, 281-293.

40. MacDonald, D. K. C., Noise and Fluctuations: An Introduction, John Wiley and Sons, New York, 1962.

41. MacDonald, N., Biological Delay Systems: Linear Stability Theory, Cambridge University Press, New York, 1989.

42. Mackey, M. C. and Nechaeva, I. G., 'Noise and stability in differential delay equations', J. Dynam. Diff. Eqns. 6, 1994, 395-426.

43. Mackey, M. C. and Nechaeva, I. G., 'Solution moment stability in stochastic differential delay equations', Phys. Rev. E 52, 1995, 3366-3376.

44. Malkiel, B. G., A Random Walk Down Wall Street, W. W. Norton \& Company, New York, 1993

45. Mazo, R. M., Brownian Motion: Fluctuation, Dynamics and Applications, Clarendon Press, Oxford, 2002.

46. Mergenthaler, K. and Enghert, R., 'Modeling the control of fixational eye movements with neurophysiological delays', Phys. Rev. Lett. 98, 2007, 138104.

47. Milton, J. and Foss, J., 'Oscillations and multistability in delayed feedback control'. In: Case Studies in Mathematical Modeling: Ecology, Physiology, and Cell Biology (H G Othmer, F R Adler, M A Lewis and J C Dallon, eds). Prentice Hall, Upper Saddle River, NJ, pp. 179-198, 1997.

48. Milton, J. G., Longtin, A., Beuter, A., Mackey, M. C. and Glass, L., 'Complex dynamics and bifurcations in neurology', J. Theoret. Biol. 138, 1989, 129-147.

49. Milton, J., Cabrera, J. L. and Ohira, T., 'Unstable dynamical systems: Delays, noise and control', Europhysics Letters, 83, 2008, 48001.

50. Milton, J., Townsend, J. L., King, M. A. and Ohita, T., 'Balancing with positive feedback: The case for discontinuous control', Phil. Trans. Royal Soc. (in press).

51. Mohammed, S.-E. A., Stochastic Functional Differential Equations, Pitman, Boston, 1984.

52. Mohammed, S.-E. A. and Scheutzow, M. K. R., 'Lyapunov exponents of linear stochastic functional differential equations. Part II. Examples and case studies', Ann. Probab. 25, 1997, 1210-1240.

53. Newell, K. M., Slobounov, S. M., Slobounova, E. S. and Molenaar, P. C. M., 'Stochastic processes in postural center-of-pressure profiles', Exp. Brain Res. 113, 1997, 158-164.

54. Niculescu, S.-I. and Gu, K., Advances in Time-Delay Systems, Springer, New York, 2004.

55. Ohira, T., 'Oscillatory correlation of delayed random walks', Phys. Rev. E 55, 1997, R1255-R1258. 
56. Ohira, T. and Milton, J., 'Delayed random walks', Phys. Rev. E 52, 1995, 3277-3280.

57. Ohira, T. and Sato, Y., 'Resonance with noise and delay', Phys. Rev. Lett. 82, 1999, 2811-2815.

58. Ohira, T., Sazuka, N., Marumo, K., Shimizu, T., Takayasu, M. and Takayasu, H., 'Predictability of currency market exchange', Physica A 308, 2002, 368-374.

59. Ohira, T. and Yamane, T., 'Delayed stochastic systems', Phys. Rev. E 61, 2000, 1247-1257.

60. Patanarapeelert, K., Frank, T. D., Friedrich, R., Beek, P. J. and Tang, I. M., 'Theoretical analysis of destablization resonances in time-delayed stochastic second-order dynamical systems and some implications for human motor control', Phys. Rev. E 73, 2006, 021901.

61. Perrin, J, Brownian Movement and Molecular Reality, Taylor \& Francis, London, 1910 .

62. Rudnick, J. and Gaspari, G., Elements of the Random Walk, Cambridge University Press, New York, 2004.

63. Santillan, M. and Mackey, M. C., 'Dynamic regulation of the tryptophan operon: A modeling study and comparison with experimental data', Proc. Natl. Acad. Sci. 98, 2001, 1364-1369.

64. Weiss, G. H., Aspects and Applications of the Random Walk, North-Holland, New York, 1994.

65. Wu, D. and Zhu, S., 'Brownian motor with time-delayed feedback', Phys. Rev. E 73, 2006, 051107.

66. Yao, W., Yu, P. and Essex, C., 'Delayed stochastic differential equation model for quiet standing', Phys. Rev. E 63, 2001, 021902.

67. Yildirim, N., Santillan, M., Horik, D. and Mackey, M. C., 'Dynamics and stability in a reduced model of the lac operon', Chaos 14, 2004, 279-292. 